\title{
BROMOFENÓIS SIMPLES RELACIONADOS AO “FLAVOR” DE ORGANISMOS MARINHOS
}

Vilma Mota da Silva, Wilson Araújo Lopes e Jailson B. de Andrade*

Instituto de Química, Universidade Federal da Bahia, Campus de Ondina, 40170-290 Salvador - BA, Brasil

Márcia C. da Cunha Veloso

Centro Federal de Educação Tecnológica da Bahia, 40300-010 Salvador - BA, Brasil

Gislaine Vieira dos Santos e Aline S. Oliveira

Instituto de Biologia, Universidade Federal da Bahia, Campus de Ondina, 40170-290 Salvador - BA, Brasil

Recebido em 18/1/06; aceito em 4/8/06; publicado na web em 19/1/07

\begin{abstract}
BROMINATED PHENOLS AS KEY FLAVOR COMPOUNDS FOUND IN MARINE ORGANISMS. The perception of the flavor is an important attribute of quality in marine fish and other seafoods, being the first and main factor of discrimination for the evaluation, later acceptance and preference of the product by the consumer. Recently, the simple bromophenols have been considered an important group of key flavor compounds occurring in a wide variety of seafood species like fishes, mollusks, crustaceans and algae. When present in high concentration, in seafood, the bromophenols produce an undesirable flavor and are associated with inferior quality. Meanwhile, when present in low concentration levels (for example $\mathrm{ng} \mathrm{g}^{-1}$ ) these compounds produce a desirable marine - or ocean-like - flavor and enhance the existing flavor in seafood. Indeed, simple bromophenols are widespread in seafood but virtually absent in freshwater fish. Herein we present a review on these flavor components found in the marine environment.
\end{abstract}

Keywords: bromophenol; flavor; marine organisms.

\section{INTRODUÇÃO}

A qualidade dos alimentos de origem marinha é determinada por uma variedade de fatores pré e pós-pesca, incluindo dieta, condições ambientais, processamento, estocagem, transporte e pode estar associada à presença de substâncias químicas. Dependendo da concentração, estas substâncias podem contribuir para melhorar ou piorar a qualidade desses alimentos, pois interferem diretamente em suas características sensoriais, tornado o "flavor" mais agradável ("on-flavor") ou desagradável ("off-flavor") ${ }^{1-4}$.

Segundo a Associação Brasileira de Normas Técnicas (ABNT) o termo "flavor" significa sabor. No entanto, de acordo com a literatura internacional, "flavor" se emprega no sentido mais amplo ou da percepção global integrada de todos os sentidos, no momento da degustação e ingestão de um alimento. A esta percepção sensorial, são somadas sensações subjetivas que estão associadas aos hábitos, aos padrões culturais e à sensibilidade de cada indivíduo (Figura 1). Assim, a análise do "flavor" deve considerar também as sensações auditivas, visuais e táteis, além das gusto-olfativas ${ }^{6,7}$.

Dentre as substâncias que podem atuar e interferir na qualidade

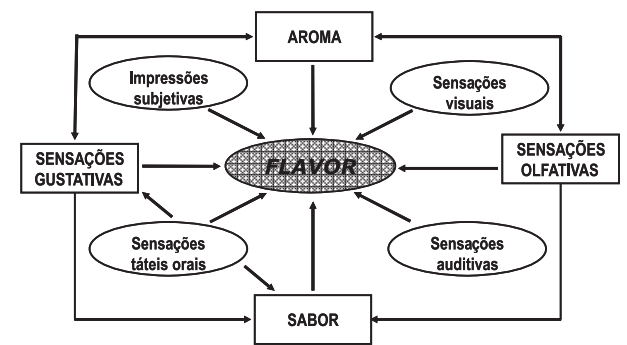

Figura 1. Esquema da inter-relação entre "flavor", sabor, aroma e as sensações gustativas e olfativas

*e-mail: jailsong@ufba.br dos alimentos de origem marinha destacam-se os bromofenóis simples, exemplificados pelo 2 e 4-bromofenol (2-BF, 4-BF), 2,4 e 2,6dibromofenol (2,4-DBF, 2,6-DBF) e 2,4,6-tribromofenol (2,4,6-TBF) ${ }^{3}$, os quais podem produzir, intensificar ou alterar o "flavor" desses alimentos. Estes bromofenóis têm sido encontrados em concentrações na ordem de ng g ${ }^{-1}$ em peixes marinhos, moluscos, crustáceos, sendo fortemente associados ao "flavor" agradável (marinado) ou desagradável (iodofórmico), em função de suas concentrações ${ }^{3,8-12}$.

A maioria dos estudos sobre o "flavor" de alimentos de origem marinha tem sido direcionada para peixes. $\mathrm{O}$ primeiro relato sobre "off-flavor" em peixes data de 1558, quando Conrad Gesner observou que o músculo do peixe Tinca tinca poderia adquirir um gosto desagradável, semelhante ao da lama. Em 1763, Bloch relatou que a carpa (Carpa oficinallis), cultivada em tanques, também adquiria um gosto similar à lama e que este "off-flavor" podia ser reduzido se o peixe fosse mantido em água limpa por algumas semanas ${ }^{13}$.

Até a primeira metade do século XX os estudos sobre "flavor" em peixes eram incipientes e restritos apenas ao estudo das características indesejáveis observadas em organismos de água doce. Alguns obstáculos contribuíram para o retardamento das investigações nessa área, destacando-se a ausência de metodologias adequadas para caracterizar e quantificar os aromas com precisão $0^{7,13,14}$.

Na década de 1960, foi observado que a ingestão de certas espécies de invertebrados pelo salmão ou pelo bacalhau produzia um "off-flavor" detectável no peixe processado ${ }^{15}$, reforçando a importância da dieta sobre o sabor dos peixes e, também, a necessidade de estudos que permitissem elucidar essa relação ${ }^{1,15,16}$. Naquela época o tema era controverso. Por ex., Stansby ${ }^{17}$, em 1962, distinguia somente dois diferentes aromas em peixes: o aroma natural, classificado como "característico", e aquele causado pela degradação.

A partir da década de 1960, as substâncias oriundas da dieta natural dos organismos marinhos passaram a ser investigadas como um dos fatores importantes para a composição do "flavor" desses alimentos. Até então, não havia associação entre sabor de peixes e substâncias químicas específicas. 
Em 1970, foi relatado que uma determinada espécie de camarão (Metapenaeus endeaveouri) pescado na Austrália apresentava um odor descrito como iodofórmico ${ }^{18}$. Somente em 1988, o "flavor" iodofórmico foi associado a substâncias halogenadas, mais especificamente ao 2,6-dibromofenol ${ }^{18,19}$.

Atualmente, a relação entre os bromofenóis e o sabor de organismos marinhos está muito bem estabelecida, sendo que a presença destes compostos é considerada de grande importância na avaliação e controle da qualidade do pescado.

O conhecimento sobre os fatores e as substâncias químicas determinantes do "flavor" certamente contribuirá para controle e melhoria de qualidade do pescado marinho.

\section{BROMOFENÓIS E O AMBIENTE MARINHO}

O ambiente marinho é considerado a maior fonte natural de compostos orgânicos halogenados. Até o início da década de 1960, as informações sobre estes compostos eram restritas aos derivados iodados da tirosina. Durante a década de 1970, aumentou consideravelmente o interesse por essa classe de substâncias, quando foram descritas mais de 300 novas substâncias. A partir da década de 1980, ocorreu um aumento significativo do número de trabalhos publicados sobre compostos orgânicos halogenados de origem marinha e, no final da década de 1990, já havia registro de cerca de 3.000 substâncias oriundas de diversas fontes naturais, sobretudo do ambiente marinho ${ }^{20-22}$.

Os estudos sobre compostos halogenados marinhos têm sido abrangentes, destacando-se os aspectos químicos relacionados particularmente à aquiicultura e, de forma geral, à química ambiental, englobando um grande número de substâncias que variam desde simples haletos orgânicos voláteis (por ex., $\mathrm{CH}_{2} \mathrm{Cl}_{2}, \mathrm{CH}_{3} \mathrm{I}, \mathrm{CHBr}_{3}$, $\mathrm{CHClBrI} \mathrm{e} \mathrm{BrCH}_{2} \mathrm{CH}_{2} \mathrm{I}$ ) até os mais sofisticados mono-, sesqui- e diterpenóides, dentre outros (Figura 2). A função biológica dessas substâncias ainda não está claramente entendida, podendo, em alguns casos, estar ligada a sistemas de defesa química. Algumas substâncias bromadas apresentam interesse farmacológico, devido às suas

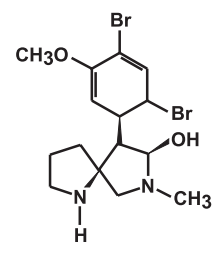

Amasthapiramida

Fonte: briozoário (Amathia wilsoni) $^{83}$
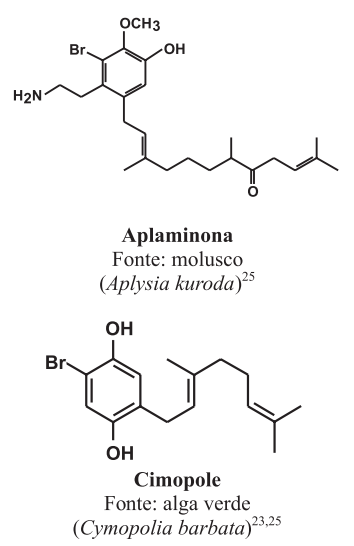

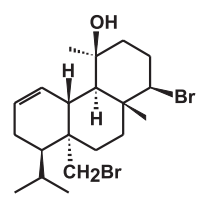

Bromosphaerol Fonte: alga vermelha

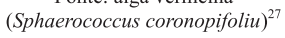

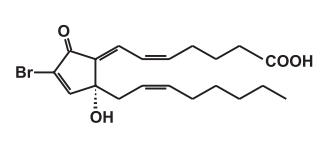

Bromovulona I Fonte: coral $(\text { Clavularia } s p)^{25}$

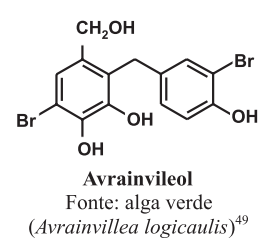

Figura 2. Exemplos de substâncias halogenadas, inclusive bromofenóis, isoladas de organismos marinhos atividades biológicas, incluindo-se, entre outras, as ações antifúngica, antibacteriana, antineoplásica, antiinflamatória e antiviral ${ }^{23-28}$.

Nos últimos anos, tem sido ampliada a atenção dedicada aos halofenóis presentes nos organismos marinhos, especificamente os bromofenóis (Figuras 2 e 3), os quais, embora possam ser considerados como poluentes antrópicos, podem também ser produzidos naturalmente por uma variedade de espécies ${ }^{20,29,30}$.

Embora exista uma grande variedade estrutural de bromofenóis comprovadamente de origem marinha, os mais estudados são o 2bromofenol, 4-bromofenol, 2,4-dibromofenol, 2,6-dibromofenol e 2,4,6-tribromofenol (Figura 3). Esses bromofenóis simples têm sido considerados como componentes principais do "flavor" característico de peixes e crustáceos marinhos, e são sintetizados a partir do bromo e de fenóis presentes no ambiente ou em organismos marinhos ${ }^{18,29,31,32}$.
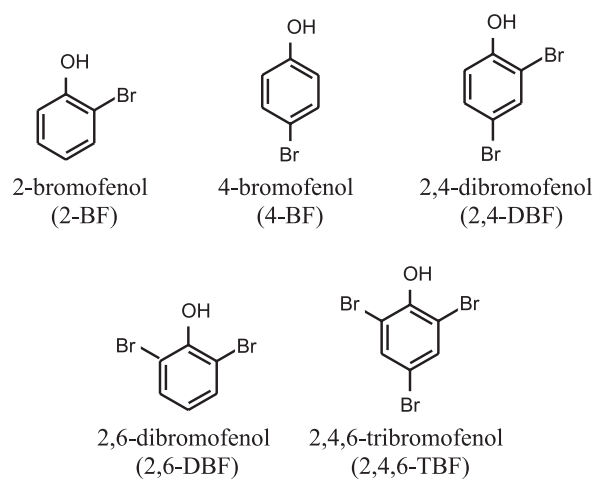

Figura 3. Bromofenóis voláteis relacionados ao "flavor" de organismos marinhos

Os bromofenóis simples estão amplamente distribuídos nos oceanos, através da cadeia alimentar. Estes compostos têm sido objetos de freqüentes investigações em espécies de peixes, crustáceos, moluscos e algas. Quando presentes em altas concentrações em peixes, os bromofenóis causam aroma desagradável ("off-flavor") e são associados à má qualidade do alimento ${ }^{18,32,33}$. Entretanto, quando presentes em baixas concentrações $\left(\mathrm{ng} \mathrm{g}^{-1}\right.$ ) são associados à melhoria do aroma e ao sabor marinado ou iodado ("on-flavor") característico das espécies de origem marinha, agregando valor ao alimento. Até o momento, os bromofenóis simples não foram observados em espé-

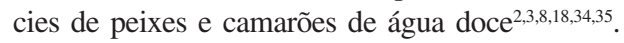

Dentre os bromofenóis simples, foi observado que o 2,6-DBF confere um "flavor" marinado ou iodofórmico à carne de lagostin, sendo considerado o mais potente, enquanto o 2-BF e 2,4,6-TBF produzem um intenso "flavor" em camarão ${ }^{1,32}$. O 2,6-DBF foi isolado em grande quantidade (10 a $15 \mathrm{mg}$ por organismo) de um hemicordado marinho, Balanoglossus biminiensis. Esse composto foi considerado o responsável pelo "flavor" iodofórmico característico desses animais e sugeriu-se que o mesmo poderia funcionar como defesa química contra predadores ${ }^{36}$.

\section{PROPRIEDADES GERAIS DOS BROMOFENÓIS SIMPLES}

Os bromofenóis simples são voláteis e muito polares. Apresentam grau variável de solubilização em água ${ }^{11}$ e solubilidade limitada em óleos vegetais. A determinação do coeficiente de partição (P) em octanol/água (expresso como log $\mathrm{P}$ ) (Tabela 1), revelou que apenas o 2,4-DBF $(\log \mathrm{P}=3,0)$ e $2,4,6-\mathrm{TBF}(\log \mathrm{P}=3,74)$ poderiam ser bioacumulados em peixes e em outros organismos marinhos. Geralmente, as substâncias com valores de $\log \mathrm{P}$ abaixo de 3,0 são solubilizadas na fase aquosa, mas não se acumulam em tecidos lipídicos ou células ${ }^{29,37-39}$. 
Em concentrações elevadas, os bromofenóis apresentam toxicidade, sendo provável que tenham alguma função defensiva contra o ataque microbiano e/ou de grandes predadores ${ }^{40,41}$. Em geral, os compostos halogenados apresentam maior toxicidade que os não halogenados ${ }^{29}$. Por ex., os fenóis monoclorados são 500 vezes mais tóxicos que o fenol e a toxicidade aumenta com o aumento do número de substituintes. $\mathrm{O}$ mesmo acontece para os derivados bromados e iodados ${ }^{29}$. Também, os compostos fenólicos clorados apresentam atividade biológica mais acentuada que os correspondentes iodofenóis e bromofenóis.

Os monoiodofenóis causam tumores de pele quando administrados em altas concentrações $\left(7.200 \mathrm{mg} \mathrm{kg}^{-1}\right)$ sobre a pele. Porém, quando administrados oralmente a ratos não são observados efeitos tóxicos ${ }^{29}$.

Os bromofenóis têm um odor desagradável, são irritantes para pele, olhos, membranas mucosas e moderamente tóxicos pela ingestão ${ }^{2,36,42,43}$. Entretanto, as concentrações de bromofenóis simples presentes naturalmente em organismos marinhos estão muito distantes daquelas que podem causar problemas toxicológicos agudos $^{2}$. Por ex., a concentração de bromofenóis em camarões australianos está na faixa de 9,5 a 1.114,0 $\mathrm{ng} \mathrm{g}^{-1} \mathrm{e}$, para que produza um efeito letal em humanos, estima-se que deveriam ser consumidos cerca de 8 milhões a 600 milhões de camarões ${ }^{29}$.

Quando administrados oralmente a roedores, as doses letais $\left(\mathrm{LD}_{50}\right)$ observadas foram: 6,52 x $10^{5} \mathrm{ng} \mathrm{g}^{-1}$ para o 2-BF; 5,3 x $10^{5} \mathrm{ng}$ $\mathrm{g}^{-1}$ para o $4-\mathrm{BF} ; 2,82 \times 10^{5} \mathrm{ng} \mathrm{g}^{-1}$ para o $2,4-\mathrm{DBF}$ e $2,0 \times 10^{6} \mathrm{ng} \mathrm{g}^{-1}$ para o $2,4,6-\mathrm{TBF}^{29}$.

O isolamento de bromofenóis de organismos marinhos envolve a extração com solventes orgânicos, geralmente utilizando técnicas especiais como a destilação-extração simultâneas (SDE), adaptada por Whitfield et $a l^{2,18}$. A separação, identificação e quantificação geralmente são feitas por técnicas de cromatografia gasosa acoplada à espectrometria de massas (CG-EM) $)^{2-3,35}$. Em estudo recente, Silva et al. $^{9}$ apresentam uma nova metodologia para isolamento, separação e identificação de bromofenóis em peixes do litoral da Bahia, Brasil, utilizando a técnica de cromatografia líquida de alta eficiência com detecção no ultravioleta (CLAE-UV).

\section{FORMAÇÃO}

Os oceanos são uma das mais importantes fontes de substâncias naturais e a ampla ocorrência de bromofenóis nesse ambiente está muito bem fundamentada. Contudo, os caminhos biossintéticos de formação dessas moléculas são um tema ainda insipiente, havendo poucos relatos na literatura que expliquem as prováveis etapas e os mecanismos das reações. Raros são os estudos biossintéticos em organismos específicos e hipóteses baseadas em relações biogenéticas isoladamente entre metabólitos. A formação de bromofenóis simples provavelmente ocorre a partir da rota do ácido chiquímico, via aminoácido tirosina ${ }^{44-46}$ (Figura 4).

Em 1975, estudando vermes marinhos, Sheikh e Djerassi ${ }^{47}$ propuseram que o 2,6-DBF e 2,4,6-TBF poderiam ser derivados da tirosina, em reações catalisadas por enzimas (Figura 4). A tirosina é convertida a ácido p-hidroxibenzóico, o qual é posteriormente bromado. A bromação ocorre após a formação do derivado do ácido benzóico, catalisada por enzimas bromoperoxidases (BPO), tais como as ferro-heme-bromoperoxidases (Fe-BPO) e as vanádiobromoperoxidases (V-BPO). Estas enzimas têm sido encontradas em todas as classes de algas marinhas, inclusive naquelas que não produzem halometabólitos, o que sugere um papel biológico extenso para as bromoperoxidases que catalisam a oxidação do brometo por peróxido de hidrogênio, produzindo o ácido hipobromoso ( $\mathrm{HBrO})$ que promove a reação de substituição eletrofílica aromática ${ }^{48-51}$.

Em estudos com vermes marinhos, Higa et al. ${ }^{42,52}$ sugeriram que a bromação ocorre através da substituição eletrofílica diretamente na tirosina, pela ação da peroxidase em presença de peróxido de hidrogênio e brometo (Figura 4). Os padrões de substituição dos derivados fenólicos formados, nas duas rotas, são compatíveis com a reação de substituição eletrofílica aromática.

Baseado em experimentos de incubação enzimática, utilizando a alga Ulva lactuca e potenciais precursores de bromofenóis (por ex., fenol, ácido 4-hidroxibenzóico, álcool 4-hidroxibenzílico, 4hidroxibenzaldeído e L-tirosina), em presença de brometo e peróxido de hidrogênio, Flodin e Whitfield ${ }^{53}$ propuseram um caminho biossintético para formação do 2,4,6-TBF.

Os experimentos com o fenol, ácido 4-hidroxibenzóico e álcool 4hidroxibenzílico produziram quantidades significativas de bromofenóis. No caso do álcool 4-hidroxibenzílico, também foram obtidos os álcoois 3-bromo-4-hidroxibenzílico e 3,5-dibromo-4-hidroxibenzílico. Quando o extrato enzimático foi incubado com o 4-hidroxibenzaldeído foi observada a formação de 3-bromo-4-hidroxibenzaldeído como produto principal da reação e pequena quantidade do derivado dibromado. No experimento com a tirosina nenhum composto bromado foi observado, demonstrando que, naquelas condições, a mesma não atuava como precursora direta de bromofenóis simples.

Com base nesses resultados e nos estudos feitos por Landymore et al. ${ }^{54}$ sobre a formação do 4-hidroxibenzaldeído, Flodin e Whitfield ${ }^{53}$ propuseram um caminho biossintético para o 2,4,6-TBF (Figura 5). Nesta rota a tirosina é transformada no ácido p-hidroxibenzóico, que é o precursor direto na formação de 2,4,6-TBF.

Os bromofenóis também podem ser formados através de processos químicos, por ex., durante a marinação de pescado (processo de

Tabela 1. Propriedades físicas de alguns bromofenóis voláteis

\begin{tabular}{|c|c|c|c|c|c|c|c|c|}
\hline Bromofenol & Aspecto físico & MM & p.f. $\left({ }^{\circ} \mathrm{C}\right)$ & p.e. $\left({ }^{\circ} \mathrm{C}\right)$ & $\mathrm{d}\left(\mathrm{g} \mathrm{cm}^{-1}\right)$ & Sol./ $\mathrm{H}_{2} \mathrm{O}(\mathrm{g} / 100 \mathrm{~mL})$ & $\log \mathrm{P}$ & Ref. \\
\hline $2-\mathrm{BF}$ & líquido oleoso & 173 & 5,6 & 194-195 & $1,492420 / 4$ & $0,22325^{\circ} \mathrm{C}$ & 1,69 & $11,37,38$ \\
\hline $4-\mathrm{BF}$ & $\begin{array}{l}\text { cristais piramidais } \\
\text { tetragonais }(\mathrm{CHCl} 3, \mathrm{Et} 2 \mathrm{O})\end{array}$ & 173 & $64-68$ & $235-236$ & $1,840^{15}$ & $1,4025^{\circ} \mathrm{C}$ & 1,94 & $11,37,38$ \\
\hline 2,4-DBF & $\begin{array}{l}\text { cristais em agulhas } \\
\text { (éter de petróleo) }\end{array}$ & 252 & $40-42^{c}$ & $238-239$ & - & $0,01225^{\circ} \mathrm{C}$ & 3,00 & 11,38 \\
\hline $2,6-\mathrm{DBF}$ & cristais em agulhas (água) & 252 & $55-57$ & $255-256$ & - & $0,1925^{\circ} \mathrm{C}$ & 2,37 & 11,38 \\
\hline $2,4,6-\mathrm{TBF}$ & $\begin{array}{l}\text { cristais longos;em } \\
\text { agulhas }(\mathrm{EtOH}) ; \\
\text { prismas (bz) }\end{array}$ & 330 & $94-96$ & $282-290$ & $2,55^{20 / 20}$ & $0,00715^{\circ} \mathrm{C}$ & 3,74 & $11,37,38$ \\
\hline
\end{tabular}

$\mathrm{MM}=$ massa molar; p.f. $=$ ponto de fusão; p.e. = ponto de ebulição; $d=$ densidade; Sol. = solubilidade

2-BF = 2-bromofenol; 4-BF = 4-bromofenol; 2,4-DBF = 2,4-dibromofenol; 2,6-DBF = 2,6-dibromofenol; 2,4,6-TBF = 2,4,6-tribromofenol; $\mathrm{CHCl}_{3}=$ clorofórmio; $\mathrm{Et}_{2} \mathrm{O}=$ éter dietílico; $\mathrm{EtOH}=$ etanol; $\mathrm{bz}=$ benzeno .

Log $\mathrm{P} \equiv$ constante de partição octanol/água. 


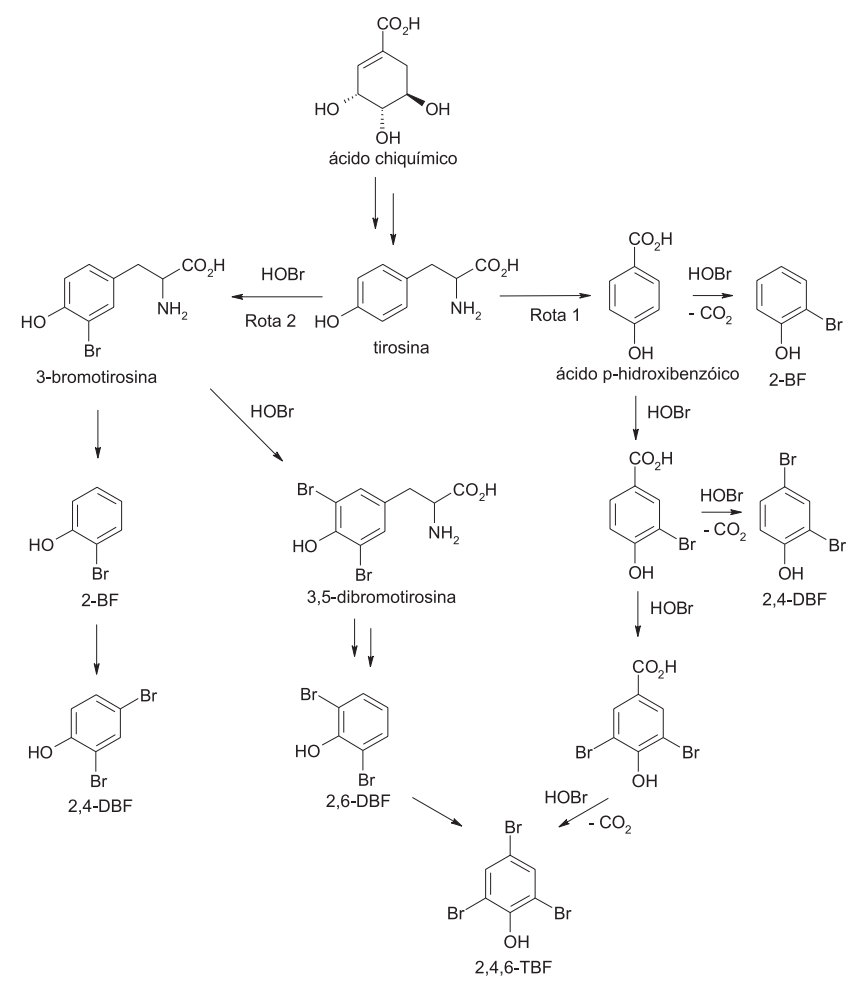

Figura 4. Esquema geral da formação dos principais precursores biogenéticos dos bromofenóis simples ${ }^{29,42}$

amaciamento e acentuação do sabor do alimento por meio da sua imersão em líquidos como vinagre, suco de limão ou vinho, junto com temperos). Também, podem ser formados durante o processo de tratamento de águas residuais submetidas à cloração, devido à presença do ion brometo. O que diferencia os dois processos é a origem dos compostos fenólicos e o mecanismo de formação do $\mathrm{HBrO}$, responsável pela bromação, através de reações de substituição eletrofílica ${ }^{29,55,56}$.

No caso da marinação, os compostos fenólicos podem ser provenientes de impurezas contidas no vinagre, enquanto que águas e sedimentos podem ser originários da degradação de compostos fenólicos complexos de organismos vivos ${ }^{29}$.

$\mathrm{O}$ mecanismo de formação do $\mathrm{HBrO}$ é distinto nos dois sistemas, devido às condições de acidez na marinação (ácida) e na cloração de águas (básico ${ }^{57}$ : na marinação o íon brometo é oxidado a $\mathrm{HBrO}$ através do peróxido de hidrogênio (Figura 6), o que

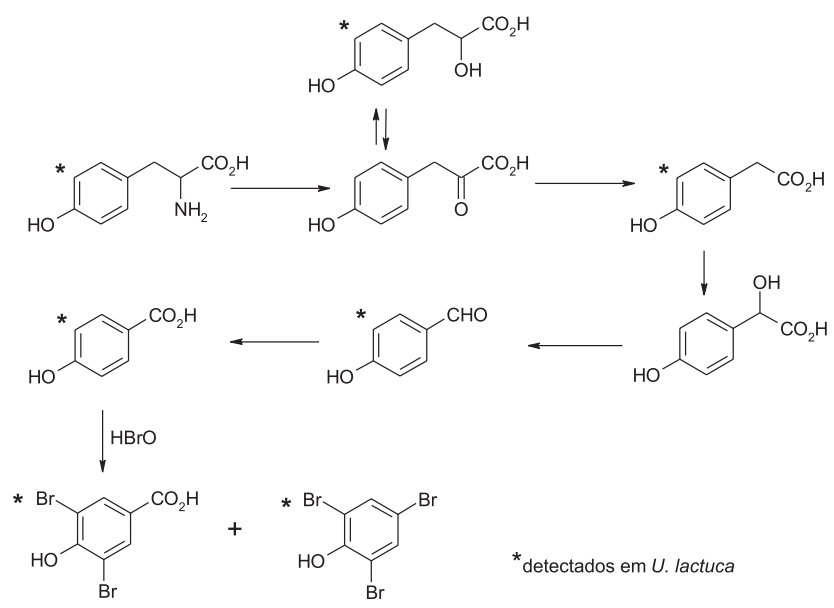

Figura 5. Proposta biossintética para a formação de 2,4,6-tribromofenol em Ulva lactuca. Adaptada da ref. 53 explica a origem dos bromofenóis encontrados em peixes que foram submetidos ao processo de marinação; no processo de cloração da água o cloro adicionado pode participar de duas reações, uma envolvendo a oxidação da água e outra, a oxidação de íons brometo. $\mathrm{O}$ bromo formado na reação reage com a água produzindo $\mathrm{HBrO}$.

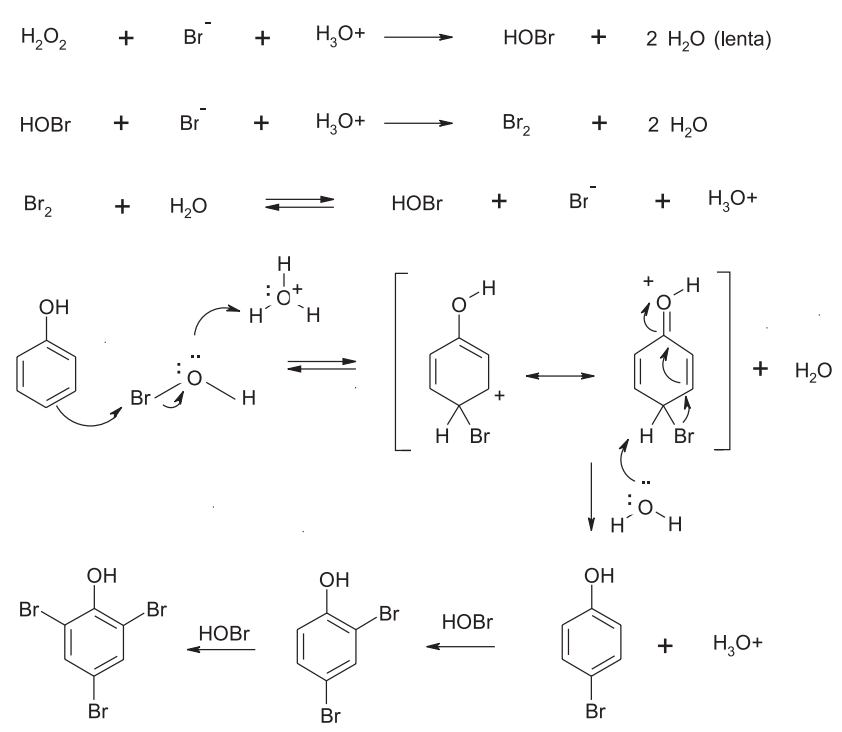

Figura 6. Mecanismo proposto para a bromação em condições ácidas. Adaptado da ref. 29

Embora o ácido hipocloroso $(\mathrm{HClO})$ formado possa competir com o $\mathrm{HBrO}$ nos processo de halogenação, a reação com este último deve predominar nas condições de $\mathrm{pH}$ (cerca de 8,3) da água do mar. Baseados nestas reações, Watanabe et al. ${ }^{58}$ sugeriram que fenóis bromados encontrados em sedimentos de rio, no Japão, foram formados como sub-produtos da cloração.

\section{OCORRÊNCIA}

As substâncias naturais bromadas de organismos marinhos foram temas de alguns artigos de revisão $0^{20,21,26,59,60}$. A maioria dos trabalhos focaliza o isolamento e a identificação de substâncias com potenciais usos farmacêuticos. Revisões anuais foram publicadas sobre substâncias bromadas complexas de ocorrência natural isoladas de organismos marinhos ${ }^{29}$. Entretanto, poucos autores têm discutido sobre as propriedades odoríferas e flavorizantes de substâncias bromadas, principalmente os bromofenóis voláteis, presentes em organismos marinhos ${ }^{1,16,29,32,36,42,61,62}$.

Os bromofenóis são produzidos diretamente por algas, briozoários e poliquetas que são classificados como produtores primários, enquanto que na maioria dos organismos marinhos, como peixes, moluscos e crustáceos, a incorporação ocorre a partir da dieta, sendo estes classificados como produtores secundários.

Os produtores primários na cadeia alimentar oceânica, como muitas espécies de algas, possuem um sistema enzimático capaz de produzir bromofenóis diretamente ${ }^{29,63}$. Ao serem consumidos, direta ou indiretamente (material orgânico resultante da decomposição de plantas ou animais marinhos), esses produtores primários transferem os bromofenóis para seus predadores ou "produtores secundários". Os produtores secundários, por sua vez, tornam-se alimentos para outros organismos marinhos e, assim, os bromofenóis são distribuídos no meio oceânico por uma complexa cadeia alimentar ${ }^{16,29,32,35,64}$.

A seguir são destacados os principais organismos marinhos produtores de bromofenóis simples. 
Algas

As algas são vegetais talófitos (plantas sem raízes, caules e folhas) fotossintetizantes, de distribuição universal, quase sempre aquáticos e, sobretudo, marinhos. Do ponto de vista econômico, são importantes fontes de insumos para a indústria farmacêutica e de cosméticos e, também, relevante fonte de alimentos para os seres humanos e animais ${ }^{65}$.

Diversas espécies de algas marinhas contêm bromofenóis simples e são capazes liberar hidrocarbonetos halogenados voláteis, tais como bromofórmio $\left(\mathrm{CHBr}_{3}\right)$, clorometano $\left(\mathrm{CH}_{3} \mathrm{Cl}\right)$, bromometano $\left(\mathrm{CH}_{3} \mathrm{Br}\right)$ e iodometano $\left(\mathrm{CH}_{3} \mathrm{I}\right)$, para a atmosfera ${ }^{53,66-73}$.

Os bromofenóis mais complexos encontrados nas algas, em sua maioria, estão na forma de sais de potássio de seus ésteres ${ }^{49}$. Até bem pouco tempo acreditava-se que os bromofenóis mais simples eram produzidos durante o processo de isolamento dessas substâncias ${ }^{74}$. Contudo, pesquisas recentes demonstram que os bromofenóis simples podem existir também em sua forma livre ${ }^{26}$.

A concentração de bromofenóis voláteis em uma grande variedade de organismos marinhos foi determinada por Whitfield et al. ${ }^{34}$, em 1992, que considerou as algas marinhas como a provável fonte primária desses compostos. Também, foi demonstrado que os bromofenóis são provenientes das dietas individuais dos peixes e que as algas marinhas são os componentes majoritários da dieta da maioria dos peixes oceânicos onívoros ${ }^{3,34}$.

Em pesquisa sobre hábitos alimentares de peixes oceânicos australianos, foram identificados nos conteúdos estomacais de 15 espécies onívoras, pelo menos sete gêneros de algas vermelhas (Rhodophyta), verdes (Chlorophyta) ou pardas (Phaeophyta), sendo que os mais comuns foram Enteromorpha, Gracilaria e Ulva ${ }^{75}$.

Em diversas espécies de algas marinhas foram identificados os bromofenóis simples 2-BF, 4-BF, 2,4-DBF, 2,6-DBF e 2,4,6-TBF e, também, demonstrado que o conteúdo total destas substâncias varia na faixa de 0,9 até $7.030 \mathrm{ng} \mathrm{g}^{-1}$, sofrendo variações sazonais ${ }^{35,75}$.

\section{Esponjas}

As esponjas são organismos bentônicos sésseis (fixos no substrato), sendo considerados animais filtradores e sedentários ${ }^{76-78}$.

Os bromofenóis voláteis 4-BF, 2,4-DBF e 2,4,6-TBF estão presentes em concentrações relativamente altas $\left(0,22-240 \mathrm{ng} \mathrm{g}^{-1}\right)$ em muitas espécies de esponjas, enquanto o 2-BF e 2,6-DBF aparecem em uma faixa de concentração significativamente mais baixa $\left(0,24-9,6 \mathrm{ng} \mathrm{g}^{-1}\right)^{34,79}$.

\section{Briozoários}

Briozoários são animais microscópicos que vivem presos a substratos como areia e rochas, sendo que a maioria vive em águas costeiras.

Todos os bromofenóis simples mais estudados são encontrados em espécies de briozoários, tais como Amathia cornuta, Bugula dentata, Cellaria pilosa ${ }^{41}$. Geralmente, o composto encontrado em maior concentração em briozoários é o 2,4,6-TBF (1.100 ng g g $\left.^{-1}\right)$, seguido por 2,4-DBF (330 ng g-1) e 2,6-DBF (210 $\left.\mathrm{ng} \mathrm{g}^{-1}\right)$.

Os briozoários são considerados como uma das principais fontes de bromofenóis para os peixes marinhos que se alimentam desses organismos. No entanto, ainda não existem relatos na literatura sobre a função dos bromofenóis nesses organismos ${ }^{3}$.

\section{Poliquetas}

Os poliquetas são vermes que desempenham um papel signifi- cativo nas cadeias alimentares marinhas, visto serem predados por peixes, crustáceos e muitos outros animais, e são descritos como potentes produtores de bromofenóis ${ }^{77-78}$. As concentrações encontradas nesses organismos são da ordem de $\mathrm{mg} \mathrm{g}^{-1}$, enquanto nas algas marinhas atingem níveis de apenas $\mathrm{ng} \mathrm{g}^{-1}{ }^{15}$. Conseqüentemente, os poliquetas são considerados como a maior fonte de bromofenol na dieta de crustáceos e peixes marinhos ${ }^{2,3,41,80}$.

As concentrações de bromofenóis nestes organismos não se correlacionam com sexo, peso ou variação sazonal. Entretanto, variações das concentrações em função do habitat e/ou de regiões geográficas são significativas, uma vez que os poliquetas que vivem em ambientes lamosos possuem maiores teores de bromofenóis que aqueles vivem em meios arenosos e rochosos ${ }^{4,81}$. Assim, não é surpreendente que espécies de peixes que se alimentam de poliquetas de ambientes lamosos contenham concentrações muito maiores de bromofenóis que aquelas que se alimentam de poliquetas de outros ambientes.

\section{Moluscos}

Os moluscos são animais predominantemente marinhos, sendo a maioria herbívora, e incluem lulas, polvos, ostras e mexilhões. Há representantes de grande importância econômica, como as muitas espécies comestíveis e as ostras que produzem pérolas.

Vários bromofenóis simples, incluindo 2,4-DBF, 2,6-DBF e 2,4,6-TBF, foram identificados em espécies de lulas e ostras. Estes compostos, além de 2-BF, 4-BF, foram também detectados em algumas espécies de moluscos (Tapes philippinarum, Ostrea rivularis $)^{11,77,78,81}$.

A presença de bromofenóis em moluscos marinhos, em seus diferentes estágios de desenvolvimento, parece estar relacionada com a dieta. Assim, as concentrações de bromofenóis mudam em decorrência da variação nas fontes alimentares. Em estudo recente, os bromofenóis foram detectados em todas as amostras de moluscos analisadas, com concentração variando na faixa de 10,3 a $246,0 \mathrm{ng} \mathrm{g}^{-181}$.

\section{Crustáceos}

Os crustáceos são animais representados por camarões, siris, caranguejos, lagostas, além de espécies da microfauna. A maioria das espécies é marinha, existindo também na água doce e salobra, havendo ainda espécies terrestres. Muitas dessas espécies são usadas na alimentação humana e são de grande importância econômica ${ }^{78}$.

Em crustáceos marinhos, os bromofenóis são considerados como sendo provenientes da dieta. Essas substâncias foram identificadas em espécies da costa australiana, Penaeus merguiensis, $P$. monodon, P. esculentus e de Hong Kong, P. japonicus, Charybdis feriatus, em concentrações de até $2.410 \mathrm{ng} \mathrm{g}^{-1}$. As concentrações variaram marcadamente entre as espécies e, também, entre as localidades onde foram coletadas ${ }^{2,18,31,81}$.

Em camarões, foi demonstrada a predominância dos bromofenóis no cefalotórax, como conseqüência da presença de resíduos alimentares no estômago. Tal fato suporta a hipótese de que esses compostos são derivados da dieta natural ${ }^{2,18,31}$. Também, foi demonstrado que camarões cultivados apresentam menor número e concentrações mais baixas de bromofenóis o que, provavelmente, pode ser explicado pelos diferentes hábitos alimentares².

A adição de bromofenóis à dieta de camarões cultivados poderia ser um recurso para modificar o "flavor", melhorar sua qualidade sensorial e despertar o interesse do consumidor. Entretanto, tentativas recentes nesse sentido têm fracassado, pois os bromofenóis perdem-se durante o preparo da ração. Assim, para serem incorporados ao alimento final, seria necessária a adição de componentes naturais à dieta (poliquetas ou algas desidratadas) ${ }^{61,82}$. 


\section{Hemicordados}

Os hemicordados são animais marinhos que, em sua maioria, vivem enterrados sob pedras, rochas e algas. Algumas espécies são conhecidas por apresentarem um "flavor" forte, caracterizado como iodofórmico ${ }^{42}$.

Em investigações feitas com as espécies Ptychodera flava, Balanoglossus carnosus, B. misakiensis e em Glossobalanus sp. foram encontrados pelo menos um dos bromofenóis simples $(2,4-$ DBF; 2,6-DBF; 2,4,6-TBF) ${ }^{42}$.

\section{Peixes}

Os peixes são animais vertebrados aquáticos, vivem em águas doce, salobra e marinha e representam uma das mais importantes fontes de alimentos para o ser humano. Além da importância nutricional, os peixes agregam qualidade à alimentação por ser considerado um alimento funcional ${ }^{77}$.

Os bromofenóis estão presentes em peixes de origem marinha e são responsáveis pelo "flavor" marinado ou iodado ("on-flavor"). A hipótese de que esses bromofenóis sejam provenientes da dieta natural é fortemente suportada pelo fato de que as concentrações desses compostos são maiores no conteúdo estomacal que aquelas encontradas no músculo ${ }^{1,3,81}$.

Observou-se que peixes carnívoros bentônicos (que se alimentam de poliquetas) e peixes herbívoros (que se alimentam de algas marinhas) apresentam um forte "flavor" marinado. Por outro lado, os peixes carnívoros piscívoros (que se alimentam de outros peixes) não apresentam o "flavor" marinado característico, provavelmente por não conterem bromofenóis ou outras substâncias halogenadas ${ }^{1,15,83}$.

Em algumas espécies de peixes as concentrações de bromofenóis dependem do habitat ${ }^{11}$. Por ex., foi observado que o salmão (Oncorhynchus sp) de origem marinha continha níveis de bromofenóis na faixa de 6,1 a 34,8 $\mathrm{ng} \mathrm{g}^{-1}$ e apresentava "flavor" marinado, enquanto o salmão de água doce não apresentava o "flavor" marinado característico e não continha bromofenóis ${ }^{1,3,15}$.

Estudos sobre a adição de algas à dieta de peixes cultivados fornecem uma base para futuras investigações sobre a incorporação e acumulação de bromofenóis, o que pode beneficiar a indústria de pescado e satisfazer o desejo dos consumidores em ter um alimento com sabor marinado ${ }^{82}$.

\section{CONSIDERAÇÕES FINAIS}

Embora exista uma grande diversidade de bromofenóis de ocorrência no ambiente marinho, nem todos estão relacionados ao "flavor" característico de determinados organismos como, principalmente, peixes e crustáceos. Entre os principais responsáveis pelo "flavor" marinado e/ou iodadado estão os bromofenóis, 2-BF, 4BF, 2,4-DBF, 2,6-DBF e 2,4,6-TBF.

Apesar dos recentes avanços, são necessárias novas investigações com objetivos específicos de: estabelecer as faixas de concentrações de bromofenóis capazes conferir o "flavor" ideal ou desejável ao pescado; identificar ou desenvolver fontes de obtenção dos bromofenóis naturais para serem incorporados às rações que servem de base à dieta de organismos cultivados e, utilizar rações contendo bromofenóis de fontes naturais no cultivo de peixes e crustáceos de água doce.

O desenvolvimento de novas tecnologias de criação de organismos aquáticos e o aprimoramento das técnicas analíticas de avaliação e controle certamente poderão contribuir para a melhoria da qualidade desses produtos. Por ser uma importante fonte alimentar para os seres humanos, pela qualidade e funcionalidade, os alimentos de origem aquática são promotores da saúde e da melhoria da qualidade de vida. Além disso, a agregação de valor a produtos destinados ao consumo interno e à exportação representa um relevante impulso na exploração comercial e industrial de pescado.

\section{AGRADECIMENTOS}

Os autores agradecem ao PRONEX/FAPESB-CNPq, CAPES e FINEP pelo suporte financeiro.

\section{REFERÊNCIAS}

1. Whitfield, F. B.; Helidoniotis, F.; Svoronos, D.; Shaw, K. J.; Ford, G. L.; Water Sci. Technol. 1995, 31, 113.

2. Whitfield, F. B.; Helidoniotis, F.; Shaw, K. J.; Svoronos, D.; J. Agric. Food Chem. 1997, 45, 4398.

3. Whitfield, F. B.; Helidoniotis, F.; Shaw, K. J.; Svoronos, D.; J. Agric. Food Chem. 1998, 46, 3750.

4. Whitfield, F. B.; Food Rev. Intern. 1990, 6, 505.

5. Associação Brasileira de Normas Técnicas; Análise Sensorial dos Alimentos e Bebidas, NBR 12806, Rio de Janeiro, 1993.

6. Fennema, O.R.; Química de los Alimentos, Editora Acribia: Zaragozaca, 1999, cap 11

7. Teixeira, E.; Meinert, E. M.; Barbetta, P. A.; Análise Sensorial de Alimentos, Editora da Universidade Federal de Santa Catarina: Florianópolis, 1987.

8. Whitfield, F. B.; Water Sci. Technol. 1999, 40, 265.

9. Silva,V. M.; Veloso, M. C. C.; Oliveira, A. S.; Santos, G. V.; Pereira, P. A. P.; de Andrade, J. B.; Talanta 2005, 68, 323.

10. Anthoni, U.; Larsen, C.; Nielsen, P. H.; Christophersen, C.; Biochem. Syst. Ecol. 1990, 18, 377.

11. Boyle, J. L.; Lindsay, R. C.; Stuiber, D. A.; J. Food Sci. 1992, 57, 4.

12. Adams, J. B.; Lock, S. J.; Toward, M. R.; Williams, B. M.; Food Chem. 1999, 64, 377

13. Persson, P. E.; Water Sci. Technol. 1995, 31, 9.

14. Stephan, A.; Bucking, M; Steinhart, H.; Food Res. Int. 2000, 33, 199.

15. Whitfield, F. B.; Helidoniotis, F.; Drew, M. Em Flavour Science: Recents Developments; Taylor, A. J.; Motram, D. S., eds.; The Royal Society of Chemistry: Cambridge, 1996.

16. Whitfield, F. B.; Water Sci. Technol. 1988, 20, 63.

17. Stansby, M. E.; Food Technol. 1962, 4, 28.

18. Whitfield, F. B.; Last, J. H.; Shaw, K. J.; Tindale, C. R.; J. Sci. Food Agric. 1988, 46, 29.

19. Baker, J. T.; Murphy, V.; Handbook of Marine Science, CRC Press: Ohio, 1976, vol. 1.

20. Gribble, G. W.; J. Nat. Prod. 1992, 55, 1353.

21. Gribble, G. W.; Acc. Chem. Res. 1998, 31, 141.

22. Gribble, G. W.; Prog. Chem. Org. Nat. Prod. 1996, 68, 1.

23. Dorta, E.; Darias, J.; San Martín, A.; Cueto, M.; J. Nat. Prod. 2002, 65, 329.

24. Jeney, E.; Zsolnai, T.; Parasitenkh. Hyg. 1967, 202, 547. (CA. 67, 4123f).

25. Neilson, A. Em The Handbook of Environmental Chemistry; Hutzinger, O. ed.; Springer-Verlag GmbH: Stockholm, 2003, vol. 3, part. R.

26. Kladi, M.; Vagias, C.; Roussis, V.; Phytochem. Rev. 2004, 3, 337.

27. Fattorusso, E.; Magno, S.; Santacroce, C.; Sica, D.; Blasio, B.; Pedone, C.; Impellizzeri, G.; Mangiafico, S.; Oriente, G.; Piatelli, M.; Sciuto, S.; Gazz. Chi. Ital. 1976, 106, 779.

28. Xu, N.; Fan, X.; Yan, X.; Li, X.; Niu, R.; Tseng, C. K.; Phytochemistry 2003, 62, 1221.

29. Boyle, J. L.; Lindsay, R. C.; Stuiber, D. A.; J. Aquat. Food Prod. Technol 1993, 2, 75 .

30. Kajiwara, T.; Kodama, K.; Hatanaka, A; Matsui, K. Em Bioctive Volatile Compounds from Plants; Teranishi, R.; Buttery, R. G.; Sugisawa, H., eds.; ASC Symposium Series: Washington, 1993, vol. 525, cap. 9.

31. Whitfield, F. B.; Shaw, K. J.; Walker, D. I.; Water Sci. Technol. 1992, 25, 131.

32. Boyle, J. L.; Lindsay, R. C.; Stuiber, D. A.; J. Aquat. Food Prod. Technol. 1992, $1,43$.

33. Höfer, T.; Water Res. 1998, 32, 3505.

34. Whitfield, F. B.; Shaw, K. J.; Svoronos, D.; $12^{\text {th }}$ International Congress of Flavours, Abstract Fragrances and Essential Oils, Vienna, Austria, 1992.

35. Chung, H. Y.; Ma, W. C. J.; Ang Jr., P. O.; Kim, J-S.; Chen, F.; J. Agric. Food Chem. 2003, 51, 2619.

36. Ashworth, R. B.; Cormier, M. J.; Science 1967, 155, 1558.

37. The Index Merck: An Encyclopedia of Chemicals Drugs and Biological, 
$10^{\text {th }}$ ed., Windholz, M., ed.;. Merck: Rahway, 1983.

38. Handbook of Chemistry and Physics: A Ready-Reference Book of Chemical and Physical Data, 54 ${ }^{\text {th }}$ ed., Weast, R. C., ed.; CRC Pres: Ohio, 1974.

39. http://www.sigmaaldrich.com, acessada em Novembro 2004.

40. Woodin, S. A.; Walla, M. D.; Lincoln, D. E.; J. Exp. Mar. Biol. Ecol. 1987, 107, 209.

41. Whitfield, F. B.; Drew, M.; Helidoniotis, F.; Svoronos, D.; J. Agric. Food Chem. 1999, 47, 4756.

42. Higa, T.; Fujiyama, T.; Scheuer, P. J.; Comp. Biochem. Physiol.,Part B: Biochem. Mol. Biol. 1980, 65, 525.

43. Lau, S. S.; Monks, T. J.; Greene, K. E.; Gillete, J. R.; Appl. Pharmacology 1984, 72, 539 .

44. Mann, J.; Secondary Metabolism, $2^{\text {nd }}$ ed., Clarendon Press: Oxford, 1987.

45. Manley, S. L.; Chapman, D. J.; Phytochemistry 1980, 19, 1453.

46. Manley, S. L.; Chapman, D. J.; FEBS Lett. 1978, 93, 97.

47. Sheikh, Y.M.; Djerassi, C.; Experientia 1975, 31, 265.

48. Butler, A.; Walker, J. V.; Chem. Rev. 1993, 93, 1937.

49. Carvalho, L. R.; Roque, N. F.; Quim. Nova 2000, 23, 757.

50. Pedersén, M.; Physiol. Plant. 1976, 37, 6.

51. Shang, M; Okuda, R. K.; Worthen, D.; Phytochemistry 1994, 37, 307.

52. Higa, T. Em Marine Natural Products; Scheuer, P.J., ed.; Academic Press: New York, 1981, vol. 4

53. Flodin, C.; Whitfield, F. B.; Phytochemistry 1999, 51, 249.

54. Landymore, A. F.; Antia, N. J.; Towers, G. H. N.; Phycologia 1978, 17, 319

55. Huston, R. C.; Ballard, M. M. Em Organic Synthesis Collective; Blatt, H., ed.; John Wiley and Sons: New York, 1943, vol. 2.

56. Pope, F. G.; Wood, A. S.; J. Chem. Soc. 1912, 101, 1823.

57. Sweetman, J. A.; Simmons, M. S.; Water Res. 1980, 14, 287.

58. Watanbe, I.; Kashimoto, T.; Tatsukawa, R.; Bull. Environ. Contam. Toxicol. 1985, 35, 272.

59. Siuda, J. F.; DeBernardis, J. F.; Lloydia 1973, 36, 107.

60. Faulkner, D. J.; Tetrahedron 1977, 33, 1421.

61. Whitfield, F. B.; Helidoniotis, F.; Smith, D.; Food Chem. 2002, 79, 355.

62. Kurata, K.; Taniguchii, K.; Takashima, K.; Hayashi, I.; SuzukI, M.; Phytochemistry 1997, 45, 485.
63. Pedersén, M.; Saenger, P.; Fries, L.; Phytochemistry 1974, 13, 2273.

64. Bemelmans, J. M. H.; den Braber, H. J. A.; Water Sci. Technol. 1983, 15, 105.

65. Oliveira, E. C.; Panorama da Aqüicultura 1997, julho/agosto, 42.

66. Fan, X.; Xu, N. J.; Shi, J. G.; J. Nat. Prod. 2003, 66, 455.

67. Fenical, W.; J. Phycol. 1975, 11, 245.

68. Fielman, K. T.; Woodin, S. A.; Walla, M. D.; Lincoln, D. E.; Mar. Ecol. Prog. Ser. 1999, 181, 1

69. Kurihara, H.; Mitani, T.; Kawabata, J.; Takahashi, K.; J. Nat. Prod. 1999, 62,882 .

70. Itoh, N.; Tsujita, M.; Ando, T.; Hisatomi, G.; Higashi, T.; Phytochemistry 1997, 45, 67 .

71. Collén, J.; Ekdahl, A.; Abrahamsson, K.; Pedersén, M.; Phytochemistry 1994, 36, 1197.

72. Nanteuil, G.; Mastagli, P.; Phytochemistry 1981, 20, 1750.

73. Ohshiro, T.; Nakano, S.; Takahashi, Y.; Suzuki, M.; IzumI, Y.; Phytochemistry 1999, 52, 1211.

74. Weinstein, B.; Rold T. L.; Harrell Jr., C. E.; Burns III, M. W.; Waaland, J. R.; Phytochemistry 1975, 14, 2667.

75. Whitfield, F. B.; Helidoniotis, F.; Shaw, K. J.; Svoronos, D.; J. Agric. Food Chem. 1999, 47, 2367.

76. http://acd.ufrj.br/labpor/1-Esponjas/Esponjas.htm., acessada em Maio 2005.

77. Rupert, E. E.; Fox, R. S.; Barnes, R. D.; Zoologia dos Invertebrados - Uma Abordagem Funcional-Evolutiva, Roca: São Paulo, 2005.

78. Hickman Jr., C. P.; Roberts, L. S.; Larson, A.; Princípios Integrados de Zoologia, 11 ${ }^{\text {a }}$ ed., Ed. Guanabara Kogan: Rio de Janeiro, 2004.

79. Hattori, T.; Konno, A.; Adachi, K.; Shiuri, Y.; Fish. Sci. 2001, 67, 899.

80. Steward, C. C.; Lovell, C. R.; Microb. Ecol. 1997, 33, 198.

81. Chung, H. Y.; Ma, W. C. J.; Kim, J-S. ; J. Agric. Food Chem. 2003, 51, 6752.

82. Ma, W. C. J.; Chung, H. Y.; Ang Jr., P. O.; Kim, J-S.; J. Agric. Food Chem. $\mathbf{2 0 0 5}, 53,2133$.

83. Whitfield, F. B.; Shaw, K. J.; Svoronos, D. Em Trends in Flavour Research; Maarse, H.; van der Heij, eds.; Amsterdam, 1994, p. 417-420. 